\title{
I NON EROI DI CARLO CASSOLA
}

\section{Giovanna Armellin Secchi}

\section{RESUMEN}

Carlo Cassola nació en Roma en 1907 y vivió en Toscana. Esta región se convirtió en el espacio donde se desarrollan sus novelas. Además, en sus trabajos se narra sobre personas comunes y sus problemas cotidianos, en un marco de problemas sociopolíticos.

\begin{abstract}
Carlo Cassola was born in Rome in 1907, and lived in Toscana. This region inspired him and not only he lived there, but also Toscana became the stage of his novels. Ordinary people and their daily problems are the aim, although sociopolitical problems are usually the frame of his literary works.
\end{abstract}

Lo scrittore italiano Carlo Cassola nasce a Roma nel 1917, ma si trasferisce in Toscana; la Maremma Toscana è il luogo ideale per inquadrare le sue opere; in vero, i paesaggi selvaggi e severi ben si addicono agli stati d'animo dei suoi personaggi, che prendono vita dal paesaggio toscano, che lo scrittore, pur romano di origine, sempre preferisce.

Nel ' 41 inizia la sua carriera letteraria, e già da allora si possono riconoscere le caratteristiche delle sue future produzioni. Si inserisce nel filone neorealista, una corrente culturale legata agli anni della guerra e del dopoguerra, però pur non sottraendosi completamente a quest'influenza, Cassola riscatta la propria originalità.

La Resistenza e i problemi socio-politici anche se sono lo sfondo di alcuni suoi racconti, non ne sono l'obiettivo principale; infatti, non è considerato uno scrittore impegnato, come pretendono gli scrittori del Neorealismo: Vittorini, Pratolini, Berto, Pavese ed altri, e quindi, da questi duramente criticato.

Si è sempre tenuto in disparte, spesso polemicamente, dalle mode letterarie dominanti, con un'indubbia, rigorosa fedeltà a se stesso e alla propria ricerca. La sua storia prende l'avvio da un'esperienza attigua all'ermetismo: dalla volontà di cogliere ed esprimere -il nudo fatto di esistere- in elementari vicende quotidiane, di là da ogni impegno ideologico, etico, psicologi$\mathrm{co}$, e anche dal gusto dell'intreccio come presentazione di eventi o caratteri privilegiati (Pazzaglia 1972: 1372). 
Cassola, in realtà, è tra i maggiori scrittori della corrente elegiaco-intimistica, anche se fa parte della letteratura della Resistenza, accetta tutti i suggerimenti del Realismo, ma li fa e li interpreta secondo la sua poetica, ciò che importa maggiormente a Cassola è l'individuo, semplice essere della vita quotidiana.

È uno scrittore attento alla dimensione umana dei personaggi, preoccupato di rispettare nelle descrizioni l'aspetto più umile e vero della realtà quotidiana. Dà, quindi, vita non a eroi, ma a delicati ritratti di persone semplici, nel loro trascorrere quotidiano, incorniciati da felicissime ricostruzioni ambientali. La misura più congeniale per quest'autore è il romanzo breve o il racconto lungo che richiamano lo spazio dell'interiorità e non quello della storia.

$\mathrm{Al}$ fondo dell'opera di Cassola c'è una sorta di malinconia esistenziale, che nasce da un deciso pessimismo storico: questa condizione trasferisce naturalmente la narrazione dal ritratto storico al disegno umano, dalla contingenza all'esistenziale (Sansone 1979: 401).

La stessa vita di Cassola orienta lo stile e l'argomento delle sue opere; durante i periodi estivi trascorsi con la familia in Toscana, la regione gli si presenta familiare e raccolta con le sue ombrose colline, i suoi boschi, i piccoli paesi, la gente umile. Lo scrittore osserva attentamente il grigiore quotidiano, tipico dell'uomo ordinario e lo descrive, e sembra registrarlo con indifferenza, ma si commuove e si illumina di poesia.

In Toscana vive anche l'esperienza partigiana e ne riconosce i suoi ideali e ne intuisce i suoi limiti, ma questo periodo matura lo scrittore che con la Resistenza conosce da vicino un'umanità nuova, la gente comune dei paesi, gli operai, gli artigiani, i contadini e i loro conflitti di coscienza, di cui parla nei suoi libri.

Da questa realtà vissuta prende spunto la tematica dei suoi racconti e romanzi: l'uomo di tutti i giorni, descritto nella sua apparente monotonia quotidiana che a volte brucia nella prova della lotta politica, dell'amore e del dolore.

L'ispirazione di Cassola si appunta programmaticamente su personaggi umili, su vicende incolori, su vite semplici e senza avventure, antiromanzesche, (...) per riprodurre il ritmo elementare del vivere (Pazzaglia 1972: 1372).

Cassola scopre la semplicità dell'esistenza in una dimensione umana, le piccole cose di ogni giorno hanno una silenziosa incidenza e possono far crollare o costruire la felicità dell'uomo.

Nel dopoguerra, Cassola inizia una serie di racconti e romanzi di fondo politico-sociale, ma soprattutto, i più riusciti di fondo sentimentale, come quello che è considerato il suo capolavoro Il taglio del bosco, ed è qui che si trova la vera natura dello scrittore.

Il taglio del bosco, che pubblica nel '50, è un romanzo autobiografico; infatti, parla di Guglielmo, un boscaiolo che, ancora giovane, rimane vedovo, come lo scrittore che è vedovo da poco quando scrive il libro.

Guglielmo, una delle figure più riuscite tra i personaggi cassoliani, è un taglialegna che nel suo faticoso lavoro tra i boschi, trova un po' di pace al dolore inconsolabile per la morte della moglie.

-Reggesse un'altra settimana- pensò dando un'occhiata indagatrice al tempo. In lui non parlava tanto l'apprensione del principale che deve corrispondere la paga anche nei giorni 
di forzata inattività e vede così scemati i guadagni. Egli paventava per se stesso quelle giornate, sapendo per esperienza come solo il lavoro valesse ad allontanare i pensieri che lo tormentavano (Cassola 1980: 69).

È padre, però incapace di quella paternità che potrebbe mitigare la sua pena; il circolo chiuso dei suoi pensieri si rinchiude sterilmente nel ricordo della moglie scomparsa. Solo il lavoro gli fa dimenticare, sia pure momentaneamente, il suo dolore, ma quando si fa sera e incupiscono i colori, una struggente tristezza invade Guglielmo e riaffiorano i dolorosi ricordi, solo temporaneamente elusi:

Poi tutti indistintamente i colori incupirono e si fusero insieme. La contentezza cadde dal cuore di Guglielmo. Rimase ancora qualche minuto lì, davanti a quell'oscura massa, mentre calava la sera. Entrando nel capanno, guardò quasi con disperazione i visi dei compagni illuminati dalla fiamma e la polenta scodellata fumante sul tavolo (Cassola 1980: 34).

Il paesaggio toscano serve da sfondo a questa tristezza: la stagione fra autunno e inverno, ormai rigida nei monti, la lunga camminata per giungere al chiaro del monte, la noia delle lunghe giornate passate nel capanno, i chiusi silenzi di Gugielmo, tutto ciò crea un'atmosfera di malinconia che riflette lo stato d'animo del protagonista.

Come è tipico nello stile di Cassola, la fedele descrizione dei dettagli dà l'impressione che la narrazione non abbia importanza, non sia determinante, però il lettore partecipa al dramma del protagonista, il dramma di una solitudine senza speranza di consolazione.

I suoi personaggi trovano invece di una ragione per cui vivere, fuori della storia, una pena esistenziale che è come predestinata, e contro la quale nulla si può. Ma tutto ciò non implica drammaticità; tutto è accettato con la consapevolezza che non c'è possibilità di ribellione contro il destino. Ė questo di più, quest'alone misterioso che circonda i gesti e le parole dei personaggi che impedisce di regolare a priori la propria vita.

Il taglio del bosco, ritenuto uno degli scritti più belli di Cassola, è il disegno di una pena pro-
fonda, assoluta, ottenuta con mezzi tecnici estremamente lineari. Le cose interiori sono dette
coi gesti, con le brevi parole neutre, coi più semplici atteggiamenti (Sansone 1979: 401).

Il fascino sottile e delicato del libro stà, appunto, nella rappresentazione degli stati d'animo dei personaggi, e specialmente del principale Guglielmo, e nella poesia largamente diffusa in tutta l'opera, soprattutto nelle descrizioni naturali.

Gli avvenimenti esteriori nel romanzo sono ridotti al minimo, quel tanto che è necessario, sempre con una delicatezza trepida, conciliando pienamente le esigenze logiche con la registrazione meticolosa di quanto nella nostra vita può avere un significato, anche se piccolo, meritevole di esser notato. Cassola è maestro nel cogliere e fermare tutte quelle impressioni, quelle sfumature di sentimenti, quegli aspetti della vita e della natura che in genere sfuggono all'osservazione. Il suo mondo è quello delle impressioni comunemente inavvertite, riuscendo a dar vita a una quantità di piccoli fatti, di particolari, di rilievi minuti. Per l'autore di Il taglio del bosco, la poesia è l'emozione di fronte a certe piccole cose, come il potere consolante del contatto con la natura. 
-È arrivata la primavera-. Fu colpito dalla giustezza dell'osservazione. Quella era veramente la prima mattinata primaverile. Aspirò profondamente l'aria, che pareva carica del profumo dei fiori. Il suo sguardo fu attratto da un' ape che ronzava intorno a un cespo di primule. Ronzando, l'animale sembrava esprimere la propria gioia per l'avvento della primavera (Cassola 1980: 88).

Lo scrittore ama narrare storie modeste, dove prevale la scelta della dimensione privata, del mondo degli affetti familiari; ed ogni riferimento viene osservato e interpretato a livello personale. Il problema sociale e politico è sempre meno importante del problema personale, e non determina mai il destino del personaggio. Non si trova nelle sue opere una ideologia ma il confronto di una persona con il mondo.

Si mise a guardare in alto. Quante stelle! Quanti mondi lontani e sconosciuti! Gli aveva detto una volta Don Mario che le stelle sono milioni di volte più grandi della Terra. Erano anch'esse abitate? C'erano anche lassù il lavoro, la sofferenza, la morte, il dolore? (Cassola 1980: 96).

Un vago senso di angoscia esce dalla serie di istantanee fatte ai personaggi cassoliani, che accettano con rassegnazione gli scacchi della vita con apparente impassibilità, nell'iñ potenza di fronte al destino segnato, ci si lascia vivere, ci si fa un'abitudine. Sulle vicende grava una fatalità, senza tuttavia che questo implichi una qualsiasi tragicità, ma un'abitudine, per cui tutto ciò che accade è subito scontato, e si affrontano gli alti e bassi della vita con compostezza e rassegnazione.

Al cancello del camposanto, non gli era mai accaduto di sentirsi così disperato, nemmeno nei giorni della disgrazia. Per qualche momento farneticò addirittura, pensava di sedersi lì in terra e lasciarsi morire. (...) Pensava che Rosa avrebbe dovuto aiutarlo. Non era possibile continuare così. Lassù dal cielo doveva dargli la forza di vivere. E guardò in alto. Ma era tutto buio, non c'era una stella (Cassola 1980: 98-9).

È la ricerca di un possibile accordo fra il mondo, in cui vivono gli uomini e il mondo assoluto, e suscita la meditazione sulla vita, sulla pena di vivere, sulla speranza.

Guglielmo è incapace di comunicarsi con i compagni di lavoro, e il vuoto si estende dolorosamente attorno a lui; si sente escluso dalla vita. Capisce la ragione dell'esistenza solo quando sa accettarsi nella sofferenza e può stabilire un dialogo umile e leale con i compagni, quando la pietà e la comprensione degli altri lo obbligano a uscire dalla sua amara solitudine.

Guglielmo guardò l'uomo e sentì improvvisamente una grande pietà per lui. Poveraccio! Esser rimasto solo al mondo, dover farsi da mangiare da sé, non avere nessuno ad assisterlo in caso di malattia. -Io mi ritengo disgraziato,-pensò -ma c'è chi sta peggio di me. Io almeno ho una sorella, ho le bambine, c'è ancora chi mi vuol bene, chi si prende cura di me.- (Cassola 1980: 95).

Le ambiguità dei silenzi, l'assommarsi di dialoghi casuali, mostra il peso di un'esistenza dura ed angosciante. Per Cassola esiste dentro di noi un mondo di affetti, di bontà, di tristezza e un gran bisogno di confidarsi, che spesso resta chiuso e non si può quasi intravedere. 
Ma la luce del giorno non era adatta alle confidenze, e così mangiarono e fecero la loro fumatina in silenzio. Nel pomeriggio Guglielmo aiutò a caricare le balle di carbone. Il mulattiere sarebbe tornato la mattina dopo per l'ultimo carico. Dopodiché, partenza! (Cassola 1980: 96).

I personaggi scontrosi e umani allo stesso tempo, che ritroviamo nel romanzo di Cassola, nascondono una conflittualità interiore, una dimensione umana, in cui si riflettono i casi e gli avvenimenti generali della storia recente, ma anche nei libri della Resistenza, alla quale partecipa attivamente, all'autore preoccupa di più la persona e la sua anima più che il paesaggio eroico.

I suoi personaggi nascono e muoiono con le loro chiuse e individualissime ragioni di esistere, solo apparentemente monotone, in realtà, proprio perché individualissime, uniche e irripetibili. Così come la misurata prosa di Cassola, così equilibratamente sfumata di toscanesimo mai manierato, rimane perennemente fedele alle sue clausule brevi, semplici, infinitamente musicali. (Babou 1972: 218).

Fra gli scrittori di ispirazione socialista, Cassola è tra i pochi che ricerca l'uomo e la sua pena. Gli uomini della Resistenza, le loro donne, i boscaioli della regione toscana, si presentano con un'umanità dolente; li vediamo nella dura esperienza quotidiana, nelle vicissitudini molto spesso dolorose, inspiegabili per lo scrittore.

Ma Cassola non penetra nell'intimo dei suoi personaggi, soltanto osserva e ne coglie le reazioni. Non vuole scavare nel profondo per scoprire le più segrete cause del loro comportamento e trovare una chiara risposta ai problemi che dà la vita; a lui basta seguirli nelle vicende di ogni giorno e descrivere la vita com'è, con i suoi momenti favorevoli e sfortunati. Cassola giunge al limite dell'inquisizione umana, però non sa o non vuole andare oltre, non fornisce una soluzione ai grandi interrogativi dell'umanità.

Cassola è fedele ai temi e ai personaggi; li riprende individualmente e minuziosamente; in successive occasioni, li presenta al lettore alla luce di una nuova esperienza individuale e sociale. I personaggi dei suoi ultimi romanzi sono più vigorosi perché alimentati da un'esperienza umana e letteraria più completa, però non molto diversi dai primi.

\begin{abstract}
All'indomani dell'ultima guerra, Cassola accetta il confronto con la realtà etico-politica contemporanea, pur restando fedele ai canoni della sua poetica. Le vicende della guerra e della Resistenza sono colte da lui nelle coscienze semplici, nei popolani umili per i quali l'ideologia e la politica sono persuasioni intimamente connesse alla loro esperienza quotidiana (Pazzaglia 1972: 1372-3).
\end{abstract}

Nel '59 si ispira alla realtà politica contemporanea con La Ragazza di Bube, dove Bube è un ex partigiano e rappresenta la delusione storica, ma al di là da ogni impegno socio-politico, il sentimento più largamente rappresentato è certo quello dell'amore. Infatti, descrive dettagliatamente l'itinerario dei sentimenti, dalla gioia alla triste attesa di Mara, la ragazza di Bube, che è veramente la principale protagonista del romanzo.

Le parole erano intermezzate dalle carezze e dagli abbracci. Finché Mara si lasciò baciare senza piú opporre resistenza. Tornarono indietro tenendosi per mano. Si guardavano, poi riabbassavano gli occhi sulle foglie, i rametti e le pallottole giallastre e pelose di cui era cosparso l'asfalto. Mara non pensava a nulla: si abbandonava tutta alla dolcezza di quel contatto, di quegli sguardi. Ma una volta in paese, tornò in lei la coscienza che non doveva abbandonarsi a quel sentimento (Cassola 1978: 195). 
In realtà, Cassola è maestro nella descrizione dei sentimenti e delle pene umane; e le sue opere più riuscite sono quelle in armonia con la natura dell'uomo, e non quelle sulla lotta partigiana. L'avventura di qualsiasi persona, anche la più sconosciuta, è degna di interesse, di preoccupazione e di pietà, perché ogni essere è come una cellula del gran tessuto della vita e contribuisce a svelarne il segreto.

Il taglio del bosco è l'emozione di fronte all'esistenza angosciata di Guglielmo, boscaiolo non eroe, e il suo problema esistenziale personale, indipendente dal problema socio-politico del momento.

In quest'opera l'autore traduce con la parola l'essenzialità della vita, considerata e raccontata nel suo profondo e quasi impenetrabile contenuto umano, e coglie l'occasione per esprimere il suo mondo interiore di ricordi, di sentimenti e di umanità.

\section{Bibliografía}

Ascor Rosa, Alberto. 1969. Scrittori e popolo. Roma: Savelli.

Babou, Victor. 1972. L'Italia del nostro tempo. Firenze: Le Monnier.

Cassola, Carlo. 1961. Un cuore arido. Torino: Einaudi.

1962. La visita. Torino: Einaudi.

1978. La ragazza di Bube. Milano: Mondadori.

1980. Il taglio del bosco. Milano: Rizzoli.

Contini, Gianfranco. 1968. Letteratura dell'Italia Unita. Firenze: Sansoni.

Pazzaglia, Mario. 1972. Gli Autori della letteratura italiana. Bologna: Zanichelli.

Pullini, Giorgio. 1965. Il romanzo del dopoguerra. Padova: Marsiglio.

Ricciardi, Mario. 1992. La letteratura in Italia. Milano: Bompiani.

Salinari, Carlo. 1976. Storia della letteratura italiana. Roma-Bari:Editori Laterza.

Sansone, Mario. 1979. Disegno storico_della letteratura italiana. Milano: Principato.

1981. Disegno storico della civiltà letteraria italiana. Firenze: Le Monnier. 